\title{
Numerical Stability of Descent Methods for Solving Linear Equations
}

\author{
Jo A.M. Bollen
}

Department of Applied Mathematics, Twente University of Technology, 7500 AE Enschede, The Netherlands

\begin{abstract}
Summary. In this paper we perform a round-off error analysis of descent methods for solving a linear system $A x=b$, where $A$ is supposed to be symmetric and positive definite. This leads to a general result on the attainable accuracy of the computed sequence $\left\{x_{i}\right\}$ when the method is performed in floating point arithmetic. The general theory is applied to the Gauss-Southwell method and the gradient method. Both methods appear to be well-behaved which means that these methods compute an approximation $x_{i}$ to the exact solution $A^{-1} b$ which is the exact solution of a slightly perturbed linear system, i.e. $(A+\delta A) x_{i}=b,\|\delta A\|$ of order $\varepsilon\|A\|$, where $\varepsilon$ is the relative machine precision and $\|\cdot\|$ denotes the spectral norm.
\end{abstract}

Subject Classifications: AMS(MOS): 65F10, 65G05; CR: J.2.

\section{Introduction}

In this paper we consider the numerical process of determining the solution $\hat{x}$ $:=A^{-1} b$ of a linear system $A x=b$ of order $n$ by a descent method (DM), where $A$ is symmetric and positive definite. The fundamental underlying structure of descent methods is as follows. Given an objective function $F(x)$, one starts at an initial point, determines, according to a fixed rule, a direction of movement and then moves in that direction to the local minimum of the objective function. At the new point a new direction of movement is determined and the process is repeated. The difference between various descent methods rests with the choice of the objective function and with the rule by which successive directions of movement are selected. We restrict ourselves to the case where the objective function is $F(x):=(\hat{x}-x, A(\hat{x}-x))$, expressed in terms of the Euclidean inner product. We only consider different choices for the directions of movement. In contrast with direct methods, like for instance Gaussian elimination, the DM's do not alter the original matrix. In fact, it is possible to avoid storing the matrix explicitly. All that is required is a subroutine that 
produces $A x$ for a given vector $x$. This is one of the main reasons why DM's became attractive for solving large sparse linear systems. Full advantage can be taken of the sparsity structure of $A$ and no assumptions need to be made about the pattern of nonzeros. Also the storage requirements are quite modest and the implementation is easy. In the case of exact computations a DM in general produces an infinite sequence $\left\{x_{i}\right\}$ whose limit is the solution $\hat{x}$. Each approximation is obtained from its predecessor by a finite number of arithmetical operations. In the presence of round-off due to floating point computations, the computed sequence $\left\{x_{i}\right\}$ not necessarily converges to $\hat{x}$. In this paper we present results on how close at least one $x_{m}$ of the computed sequence $\left\{x_{i}\right\}$ of a DM approaches the solution $\hat{x}$ (referred to as attainable accuracy). Some wellknown DM's are Gauss-Seidel, Gauss-Southwell, gradient (or steepest descent) and conjugate gradient method. The results presented in this paper are a generalization of the results presented in [1], concerning the gradient method. Other iterative methods like Jacobi, Richardson, SOR and Chebyshev iterations have been investigated by Woźniakowski in recent years (cf. [4] and [5]). We also present results concerning the speed of convergence of DM's in the presence of round-off.

In this paper $(\cdot, \cdot)$ stands for the Euclidean inner product, $\|\cdot\|$ in connection with a vector stands for the Euclidean norm and $\|\cdot\|$ in connection with a matrix stands for the spectral norm. $\kappa$ denotes the condition number $\|A\|\left\|A^{-1}\right\|$ of the symmetric positive definite matrix $A$. The matrices $A^{\frac{1}{2}}, A^{-\frac{1}{2}}$ are the uniquely determined symmetric positive definite matrices satisfying $A^{\frac{1}{2}} A^{\frac{1}{2}}=A$ and $A^{-\frac{1}{2}}=\left(A^{\frac{1}{2}}\right)^{-1}$. Note that the following relations hold for $\alpha=\frac{1}{2}$ and $\alpha=-\frac{1}{2}:\left(x, A^{2 \alpha} x\right)=\left(A^{\alpha} x, A^{\alpha} x\right)=\left\|A^{\alpha} x\right\|^{2}$.

Given a definite system $A x=b$ and a sequence of arbitrary nonzero vectors $\left\{p_{i}\right\}$ then the corresponding DM is defined by the following statements.

Descent Method (DM)

Choose an initial point $x_{0}$;

while $r_{i} \neq 0$ do begin

$$
\begin{aligned}
r_{0} & :=b-A x_{0} ; \quad i:=0 ; \\
a_{i} & :=\left(r_{i}, p_{i}\right) /\left(p_{i}, A p_{i}\right) ; \\
x_{i+1} & :=x_{i}+a_{i} p_{i} ; \\
r_{i+1} & :=b-A x_{i+1} ; \\
i & :=i+1
\end{aligned}
$$

end.

There is no need to know the direction vectors $p_{i}$ in advance, they can as well be determined as the process proceeds. The case where the computations are carried out exactly is referred to as an exact DM and the case where the computations are carried out in floating point arithmetic is referred to as a perturbed DM. We often use a circumflex to distinguish between situations where exact as well as computed quantities are involved; non-circumflexed Latin letters denote machine quantities (e.g. $r_{i}, \hat{r}_{i}, a_{i}, \hat{a}_{i}$ ). 
We summarize the contents of the paper. In section 2 we deduce some wellknown algebraic properties of exact DM's which are fundamental for studying some properties of perturbed DM's. In subsection 3.1 we present some preliminaries, basic tools and notions needed for our round-off error analysis in the following (sub)sections. In subsection 3.2 we derive our main theorem which contains an analogue of the algebraic properties of section 2 for the perturbed DM's. In subsection 4.1 and 4.2 we sucessively apply this main theorem to the Gauss-Southwell method (GSM) and the gradient method (GM). Results concerning the conjugate gradient method can be found in Bollen [2]. In Sect. 5 we give final remarks.

\section{The Exact DM}

In this section we consider general exact DM's, defined by the algorithm in Sect. 1. Some well-known elementary properties are stated in the following theorem.

Theorem 2.1. At each step an exact DM minimizes the objective function.

$$
F(x):=((\hat{x}-x), A(\hat{x}-x))=\left\|A^{\frac{1}{2}}(\hat{x}-x)\right\|^{2}
$$

along the line $x=x_{i}+a p_{i}$ and

where

$$
\frac{\left\|A^{\frac{1}{2}}\left(\hat{x}-x_{i+1}\right)\right\|^{2}}{\left\|A^{\frac{1}{2}}\left(\hat{x}-x_{i}\right)\right\|^{2}}=1-\gamma_{i}^{2},
$$

$$
\gamma_{i}:=\frac{\left|\left(r_{i}, p_{i}\right)\right|}{\left\|A^{-\frac{1}{2}} r_{i}\right\|\left\|A^{\frac{1}{2}} p_{i}\right\|} .
$$

Furthermore

and

$$
\left\|A^{-\frac{1}{2}} r_{i+1}\right\|^{2}+\left\|a_{i} A^{\frac{1}{2}} p_{i}\right\|^{2}=\left\|A^{-\frac{1}{2}} r_{i}\right\|^{2}
$$

Proof. Along the line $x=x_{i}+a p_{i}$ we have

$$
\begin{aligned}
F(x) & =\left\|A^{\frac{1}{2}}\left(\hat{x}-x_{i}-a p_{i}\right)\right\|^{2}=F\left(x_{i}\right)-2 a\left(A\left(\hat{x}-x_{i}\right), p_{i}\right)+a^{2}\left\|A^{\frac{1}{2}} p_{i}\right\|^{2} \\
& =F\left(x_{i}\right)+\left\|A^{\frac{1}{2}} p_{i}\right\|^{2}\left(a-\frac{\left(A\left(\hat{x}-x_{i}\right), p_{i}\right)}{\left\|A^{\frac{1}{2}} p_{i}\right\|^{2}}\right)^{2}-\frac{\left(A\left(\hat{x}-x_{i}\right), p_{i}\right)^{2}}{\left\|A^{\frac{1}{2}} p_{i}\right\|^{2}},
\end{aligned}
$$

which is minimal for

$$
a=\frac{\left(A\left(\hat{x}-x_{i}\right), p_{i}\right)}{\left\|A^{\frac{1}{2}} p_{i}\right\|^{2}}=\frac{\left(r_{i}, p_{i}\right)}{\left(p_{i}, A p_{i}\right)}=a_{i}
$$

and the minimal value $F\left(x_{i}+a_{i} p_{i}\right)=\left\|A^{\frac{1}{2}}\left(\hat{x}-x_{i+1}\right)\right\|^{2}$ satisfies

$$
\left\|A^{\frac{1}{2}}\left(\hat{x}-x_{i+1}\right)\right\|^{2}=\left\|A^{\frac{1}{2}}\left(\hat{x}-x_{i}\right)\right\|^{2}-\left(r_{i}, p_{i}\right)^{2} /\left\|A^{\frac{1}{2}} p_{i}\right\|^{2} .
$$


Since $A^{\frac{1}{2}}\left(\hat{x}-x_{i}\right)=A^{-\frac{1}{2}}\left(A \hat{x}-A x_{i}\right)=A^{-\frac{1}{2}} r_{i}$ and $\left(r_{i}, p_{i}\right)^{2} /\left\|A^{\frac{1}{2}} p_{i}\right\|^{2}=\left\|a_{i} A^{\frac{1}{2}} p_{i}\right\|^{2}$, formulas (2.2) and (2.4) follow readily from (2.6). Using

$$
r_{i+1}=b-A x_{i+1}=b-A x_{i}-a_{i} A p_{i}=r_{i}-a_{i} A p_{i}
$$

we obtain, from the definition of $a_{i}$,

which proves (2.5).

$$
\left(r_{i+1}, p_{i}\right)=\left(r_{i}, p_{i}\right)-a_{i}\left(p_{i}, A p_{i}\right)=0,
$$

As a direct consequence of Theorem 2.1 it follows that if $\left\{x_{i}\right\}$ is generated by an exact DM and if $\gamma_{i}$ is uniformly bounded away from zero, say $\gamma_{i} \geqq \gamma>0$ for all $i \geqq 0$, then we have, for all $i \geqq 0$,

$$
\frac{\left\|A^{\frac{1}{2}}\left(\hat{x}-x_{i+1}\right)\right\|^{2}}{\left\|A^{\frac{1}{2}}\left(\hat{x}-x_{i}\right)\right\|^{2}}=\frac{\left\|A^{-\frac{1}{2}} r_{i+1}\right\|^{2}}{\left\|A^{-\frac{1}{2}} r_{i}\right\|^{2}} \leqq 1-\gamma^{2} .
$$

This mean that in that case, for the exact DM, the so-called natural error $\left\|A^{\frac{1}{2}}\left(\hat{x}-x_{i}\right)\right\|$ converges step-wise linearly to zero with a convergence ratio no greater than $\left(1-\gamma^{2}\right)^{\frac{1}{2}}$. The parameter $\gamma_{i}$ depends on the choice of the direction vector $p_{i}$. For most of the commonly used DM's the parameter $\gamma_{i}$ is uniformly bounded away from zero. Obviously, always $\gamma_{i} \leqq 1$. In our numerical analysis we often use, instead of the parameter $\gamma_{i}$, the related parameters $\alpha_{i}$ and $\beta_{i}$ defined by

$$
\alpha_{i}:=\frac{\left\|r_{i}\right\|\left\|p_{i}\right\|}{\left|\left(r_{i}, p_{i}\right)\right|}, \quad \beta_{i}:=\frac{\left\|r_{i}\right\|\left\|A^{\frac{1}{2}} p_{i}\right\|}{\left\|A^{\frac{1}{2}}\right\|\left|\left(r_{i}, p_{i}\right)\right|},
$$

assuming that $\left(r_{i}, p_{i}\right) \neq 0$. Note that from the Cauchy-Schwarz inequality it follows that

$$
\kappa^{-\frac{1}{2}} \leqq \kappa^{-\frac{1}{2}} \alpha_{i} \leqq \beta_{i} \leqq \gamma_{i}^{-1} \leqq \kappa^{\frac{1}{2}} \beta_{i} \leqq \kappa^{\frac{1}{2}} \alpha_{i},
$$

which relates upper and lower bounds of $\alpha_{i}$ and $\beta_{i}$ on one hand and lower and upper bounds of $\gamma_{i}$ on the other hand.

\section{The Perturbed DM}

In this section we consider general perturbed DM's for the case where the statements of the algorithm in Sect. 1 are performed using floating point arithmetic.

\subsection{Notations, Definitions and Conventions}

Rounding Errors. We assume that the DM-algorithm given in Sect. 1 is performed on a floating point machine with relative precision $\varepsilon$ and that adding or subtracting two machine vectors $x$ and $y$ and multiplying a machine vector $x$ and a machine number $a$ yield computed vectors $\mathrm{fl}(x \pm y)$ and $\mathrm{fl}(a x)$ satisfying

$$
\begin{aligned}
\mathrm{fl}(x \pm y) & =\left(I+F_{1}\right)(x \pm y), & & \left\|F_{1}\right\| \leqq \varepsilon, \\
\mathrm{fl}(a x) & =\left(I+F_{2}\right)(a x), & & \left\|F_{2}\right\| \leqq \varepsilon .
\end{aligned}
$$


Note that (3.1) is fulfilled in all practical implementations, where $F_{1}$ and $F_{2}$ are diagonal matrices. Furthermore we assume that the matrix by vector product calculation of a machine matrix $A$ and a machine vector $x$ and the inner product calculation of two machine vectors $x$ and $y$ satisfy the relations

$$
\begin{array}{rlrl}
\mathrm{fl}(A x) & =(A+E) x, & & \|E\| \leqq \varepsilon C_{1}, \\
\mathrm{fl}((x, y)) & =((I+D) x, y), & \|D\| \leqq \varepsilon C_{2} .
\end{array}
$$

where $C_{1}$ and $C_{2}$ are constants depending only on $n$ and $\varepsilon$. Throughout this paper $C_{1}$ and $C_{2}$ stand for the upper bounds of the round-off matrices $E$ and $D$ according to (3.2). For the standard algorithms $E$ is a full matrix and $D$ is a diagonal matrix whereas $C_{1}$ is of order $n^{3 / 2}$ and $C_{2}$ is of order $n$. In our roundoff error analysis we neglect the possibility of underflow and overflow. We assume that in appropriate expressions none of the exact or computed denominators equals zero.

o-notation. In order to simplify the expressions arising from the application of the basic relations (3.1) and (3.2) we use a kind of Bachmann-Landau o-notation to be able to neglect terms of order $\varepsilon^{2}$ in the presence of a term of order $\varepsilon$, with a minimal loss of relevant information. For instance, we write

$$
\varepsilon\left(1+C_{1} \kappa+2 \varepsilon C_{2} \kappa^{\frac{1}{2}}\right)=\varepsilon\left(1+C_{1} \kappa+o\right), \quad\left[\varepsilon C_{2} \kappa^{\frac{1}{2}} \rightarrow 0\right],
$$

where the expression between square brackets indicates that $o$ stands for a quantity which is smaller than a constant times $\varepsilon C_{2} \kappa^{\frac{1}{2}}$. The formal definition of the $o$-symbol reads as follows.

Definition 3.1. Let $f$ and $g$ be two scalar functions defined on a set $R \subseteq \mathbb{R}^{l}$ $(l \in \mathbb{N})$. Then $f(x)=o,[g(x) \rightarrow 0]$ means that there exist constants $K>0, \delta>0$ such that $|f(x)| \leqq K|g(x)|$ for all $x \in R$ satisfying $|g(x)| \leqq \delta$.

In this paper $K$ and $\delta$ are supposed to be numerical constants, not depending on $\varepsilon, \kappa, C_{1}$ and $C_{2}$. The expression between square brackets $[g(x) \rightarrow 0]$ is referred to as the restriction under which $f(x)=o$ holds. The symbol $o$ only supplies information to those $x$ for which $g(x)$ is small. We give the interpretation of some equalities and inequalities containing the $o$-symbol. If we write, for two scalar functions $f_{1}$ and $f_{2}$, with $f_{2}(x)>0 \quad(x \in R), f_{1}(x) \leqq f_{2}(x)$ $(1+o),[g(x) \rightarrow 0]$, then we mean that there exists a scalar function $f_{3}$, defined on $R$, such that $\left(f_{1}(x)-f_{2}(x)\right) / f_{2}(x) \leqq f_{3}(x)$ on $R$ and $f_{3}(x)=o$, $[g(x) \rightarrow 0]$. If the $o$ symbol appears in some compound formula or at both sides of an equality or inequality relation, then the $o$-symbol has to be interpreted as a class of functions. For instance, if we write $f_{1}(x) o=o,[g(x) \rightarrow 0]$, then this has to be interpreted as follows. For any function $f_{2}$ for which $f_{2}(x)=o,[g(x) \rightarrow 0]$, one also has $f_{1}(x) f_{2}(x)=o,[g(x) \rightarrow 0]$. Four rather trivial but often used properties are $g(x)=o, o+o=o, o o=o$ and $(1+o)^{-1}=1+o$, all holding under the restriction $[g(x) \rightarrow 0]$. These properties indicate that the $o$-symbol is easy to handle and that is our main reason for using it. A disadvantage of the use of $o$ symbols is that we do not obtain explicit numerical constants in error estimates. However, in all cases where we derive formulas with $o$-symbols it is possible to retrace the proof, replacing all $o$-symbols by estimates involving explicit numerical constants. That is, at every stage of the proof we are able to 
indicate definite numbers, where the asymptotic estimates only state the existence of such numbers (cf. formulas (3.2) and (3.33)). However, these definite numbers are rather arbitrary, whereas the coefficients in the equality relations involving $o$-symbols are more or less uniquely determined (compare e.g.

$$
\begin{aligned}
& 1+\varepsilon \leqq(1-\varepsilon)^{-1} \leqq 1+(1+1 / 3) \varepsilon, \quad[0<\varepsilon<1 / 4] \quad \text { and } \\
& \left.(1-\varepsilon)^{-1}=1+(1+o) \varepsilon, \quad[\varepsilon \rightarrow 0]\right) .
\end{aligned}
$$

Good-Behavior and Numerical Stability. To denote the quality of the approximate solution computed by an iterative method with floating point arithmetic we use the concepts of good-behavior and numerical stability (cf. Woźniakowski $[4,5])$.

Definition 3.2. An iterative method for solving a linear system $A x=b$ is said to be well-behaved (or, equivalently, has good-behavior) if for all initial points $x_{0}$ the computed sequence $\left\{x_{i}\right\}$ contains at least one approximation $x_{m}$ such that, for some matrix $\delta A,(A+\delta A) x_{m}=b$, and $\|\delta A\| \leqq g \varepsilon\|A\|$, where $g$ depends only on the dimension of the system.

Good-behavior means that the computed approximate solution $x_{m}$ is the exact solution of a slightly perturbed system. From a practical point of view, this solution $x_{m}$ is satisfactory since the elements of the machine matrix $A$ itself in general cannot be a better representation of the elements of the original matrix than with relative precision $\varepsilon$. Therefore, the corresponding error in $b-A x_{m}$ is inherent for the system $A x=b$ (cf. Stoer and Bulirsch [3]). It is easy to verify that good-behavior is equivalent to the assertion that the residual $\| A\left(\hat{x}-x_{m} \|\right.$ satisfies

$$
\| A\left(\hat{x}-x_{m}\|\leqq g \varepsilon\| A\|\| x_{m} \| .\right.
$$

Therefore $\varepsilon\|A\|\left\|x_{m}\right\|$ is called the inherent residual. It can also easily be seen that (3.3) implies $\left\|A^{\frac{1}{2}}\left(\hat{x}-x_{m}\right)\right\| \leqq g \varepsilon \kappa^{\frac{1}{2}}\left\|A^{\frac{1}{2}}\right\|\left\|x_{m}\right\|$ which, in turn, implies $\left\|\hat{x}-x_{m}\right\| \leqq g \varepsilon \kappa\left\|x_{m}\right\|$, but the implications do not hold in general vice versa. So, if $x_{m}$ is the exact solution of a slightly perturbed system in the sense of definition 3.2 , then the error $\left\|\hat{x}-x_{m}\right\|$ can be of order $\varepsilon \kappa\left\|x_{m}\right\|$ and therefore this is called the inherent error. For similar reasons $\varepsilon \kappa^{\frac{1}{2}}\left\|A^{\frac{1}{2}}\right\|\left\|x_{m}\right\|$ is called the inherent natural error. An iterative method that computes, for all initial points $x_{0}$, an approximation $x_{m}$ whose error $\left\|\hat{x}-x_{m}\right\|$ is at most of the order of the inherent error is called numerically stable (cf. Woźniakowski $[4,5])$. Thus a well-behaved method certainly is numerically stable but the reverse is not necessarily true.

\subsection{The Main Theorem}

Before starting off the round-off error analysis of a perturbed DM we first deduce some auxiliary results concerning the computation of a residual vector $b-A x$. These results will be used in subsequent considerations.

Lemma 3.1. Let $b, x$ be two machine vectors and let

$$
\begin{aligned}
\hat{r} & =b-A x, & r & =\mathrm{fl}(b-\mathrm{fl}(A x)), \\
\phi: & =\|A\|\|x\| /\|\hat{r}\|, & \psi: & =\left\|A^{\frac{1}{2}}\right\|\|x\| /\left\|A^{-\frac{1}{2}} \hat{r}\right\| .
\end{aligned}
$$


Then we have

$$
\begin{array}{cl}
(\hat{r}, r)=\|\hat{r}\|^{2}(1+o)=\|r\|^{2}(1+o), & {\left[\varepsilon\left(1+C_{1} \phi\right) \rightarrow 0\right],} \\
\left(\hat{r}, A^{-1} r\right)=\left\|A^{-\frac{1}{2}} \hat{r}\right\|^{2}(1+o)=\left\|A^{-\frac{1}{2}} r\right\|^{2}(1+o), & {\left[\varepsilon k^{\frac{1}{2}}\left(1+C_{1} \psi\right) \rightarrow 0\right],}
\end{array}
$$

Proof. According to (3.1) and (3.2) we have

$$
\begin{aligned}
r & =\mathrm{fl}(b-\mathrm{fl}(A x))=(I+F)(b-(A+E) x)=\hat{r}+\delta r, \\
\delta r & =F(b-A x)-(I+F) E x .
\end{aligned}
$$

Consequently,

$$
\|\delta r\| \leqq \varepsilon\|\hat{r}\|+\varepsilon(1+\varepsilon) C_{1}\|A\|\|x\|
$$

and hence, under the restriction $\varepsilon \rightarrow 0$,

$$
\begin{aligned}
\|\delta r\| /\|\hat{r}\| & \leqq \varepsilon\left(1+C_{1} \phi(1+o)\right), \\
\left\|A^{-\frac{1}{2}} \delta r\right\| /\left\|A^{-\frac{1}{2}} \hat{r}\right\| & \leqq \varepsilon \kappa^{\frac{1}{2}}\left(1+C_{1} \psi(1+o)\right),
\end{aligned}
$$

which can be weakened to

$$
\begin{aligned}
\|\delta r\| /\|\hat{r}\| & =o, & & {\left[\varepsilon\left(1+C_{1} \phi\right) \rightarrow 0\right], } \\
\left\|A^{-\frac{1}{2}} \delta r\right\| /\left\|A^{-\frac{1}{2}} \hat{r}\right\| & =o, & & {\left[\varepsilon K^{\frac{3}{2}}\left(1+C_{1} \psi\right) \rightarrow 0\right] . }
\end{aligned}
$$

The first equalities in (3.5) and (3.6) follow immediately from (3.7) and the appropriate equality of (3.9). The second equatities follow from the fact that for $l=0,-\frac{1}{2}$ we have

$$
\left|\left\|A^{l} r\right\|-\left\|A^{l} \hat{r}\right\|\right| \leqq\left\|A^{l} \delta r\right\| \leqq\left\|A^{l} \hat{r}\right\| o,
$$

under the appropriate restriction.

In the case of perturbed DM's we mean by $r_{i}$ always the computed residual $\mathrm{fl}\left(b-\mathrm{fl}\left(A x_{i}\right)\right)$ and by $\hat{r}_{i}$ always the exact residual $b-A x_{i}$. In the case of exact computations of course $r_{i}=\hat{r}_{i}$.

We are now ready to deduce the main theorem where the influence of round-off on relation (2.2) is expressed in terms of a relative error.

Theorem 3.1. Let $x_{i}, p_{i}$ be two arbitrary machine vectors $\left(p_{i} \neq 0\right)$ and let $x_{i+1}$ be computed from one step perturbed DM, based on these two vectors. Let

$$
\begin{aligned}
\hat{r}_{i} & =b-A x_{i}, \\
\gamma_{i}: & =\mid\left(\hat{r}_{i}, p_{i}\right) \| /\left(\left\|A^{-\frac{1}{2}} \hat{r}_{i}\right\|\left\|A^{\frac{1}{2}} p_{i}\right\|\right), \\
\alpha_{i}: & =\left\|\hat{r}_{i}\right\|\left\|p_{i}\right\| /\left(\hat{r}_{i}, p_{i}\right) \mid \\
\beta_{i}: & =\left\|\hat{r}_{i}\right\|\left\|A^{\frac{1}{2}} p_{i}\right\| /\left(\left\|A^{\frac{1}{2}}\right\| \mid\left(\hat{r}_{i}, p_{i}\right) \|\right) \\
\phi_{i}: & =\|A\|\left\|x_{i}\right\| /\left\|\hat{r}_{i}\right\| .
\end{aligned}
$$

Then we have

$$
\frac{\left\|A^{\frac{1}{2}}\left(\hat{x}-x_{i+1}\right)\right\|^{2}}{\left\|A^{\frac{1}{2}}\left(\hat{x}-x_{i}\right)\right\|^{2}}=1-\gamma_{i}^{2}\left(1+v_{i+1}\right),
$$

where

$$
\begin{aligned}
\left|v_{i+1}\right| \leqq & 2 \varepsilon\left\{2 \kappa^{\frac{1}{2}}+2 \alpha_{i}+\beta_{i}\left(1+\beta_{i}\right) \phi_{i}\right\}(1+o) \\
& +\varepsilon\left\{\left(C_{2} \kappa^{\frac{1}{2}}+C_{1} \kappa\right)+\alpha_{i} C_{2}+C_{1} \alpha_{i} \phi_{i}\right\} o
\end{aligned}
$$


under the restriction

$$
\varepsilon\left\{\kappa^{\frac{1}{2}}\left(1+C_{2}+C_{1} \kappa^{\frac{1}{2}}\right)+\alpha_{i}\left(1+C_{2}\right)+\left(\beta_{i}+C_{1} \alpha_{i}\right) \phi_{i}\right\} \rightarrow 0 .
$$

Proof. From (3.7) and (3.8) we know that the computed vector $r_{i}=\mathrm{fl}\left(b-\mathrm{fl}\left(A x_{i}\right)\right)$ satisfies

$$
\begin{aligned}
r_{i} & =\hat{r}_{i}+\delta r_{i} \\
\left\|\delta r_{i}\right\| /\left\|\hat{r}_{i}\right\| & \leqq \varepsilon\left(1+C_{1} \phi_{i}(1+o)\right), \quad[\varepsilon \rightarrow 0] .
\end{aligned}
$$

We consider the computation of $a_{i}$ from (1.1). For the numerator we obtain

$$
\begin{aligned}
\mathrm{fl}\left(\left(r_{i}, p_{i}\right)\right) & =\left(\left(I+D_{i}^{\prime}\right) r_{i}, p_{i}\right)=\left(r_{i}, p_{i}\right)\left(1+\lambda_{i}\right), \\
\left|\lambda_{i}\right| & =\left|\left(D_{i}^{\prime} r_{i}, p_{i}\right) /\left(r_{i}, p_{i}\right)\right| \leqq \varepsilon C_{2} \alpha_{i}^{\prime}, \\
\alpha_{i}^{\prime} & =\left\|r_{i}\right\|\left\|p_{i}\right\| /\left|\left(r_{i}, p_{i}\right)\right|
\end{aligned}
$$

We want to have expressions in terms of $\left(\hat{r}_{i}, p_{i}\right)$ instead of $\left(r_{i}, p_{i}\right)$. From (3.14) we obtain, under the restriction $\varepsilon \rightarrow 0$,

$$
\begin{aligned}
\left(r_{i}, p_{i}\right) & =\left(\hat{r}_{i}, p_{i}\right)\left(1+\tau_{i}\right), \\
\left|\tau_{i}\right| & =\left|\left(\delta r_{i}, p_{i}\right) /\left(\hat{r}_{i}, p_{i}\right)\right| \leqq\left(\left\|\delta r_{i}\right\| /\left\|\hat{r}_{i}\right\|\right) \alpha_{i} \\
& \leqq \varepsilon \alpha_{i}+\varepsilon C_{1} \alpha_{i} \phi_{i}(1+o) .
\end{aligned}
$$

Hence, $\left|\tau_{i}\right|=o$ under the restriction

$$
\varepsilon \alpha_{i}+\varepsilon C_{1} \alpha_{i} \phi_{i} \rightarrow 0
$$

Since $\alpha_{i} \geqq 1$ this restriction contains the restriction $\varepsilon\left(1+C_{1} \phi_{i}\right) \rightarrow 0$, so that from (3.15) and (3.16) we obtain $\alpha_{i}^{\prime}=\alpha_{i}(1+o)$ under the restriction (3.17). Consequently, under the restriction (3.17),

$$
\begin{aligned}
\mathrm{fl}\left(\left(r_{i}, p_{i}\right)\right) & =\left(\hat{r}_{i}, p_{i}\right)\left(1+\eta_{i}\right), \\
\left|\eta_{i}\right| & =\left|\lambda_{i}\left(1+\tau_{i}\right)+\tau_{i}\right| \leqq \varepsilon \alpha_{i}\left(1+C_{2}+C_{1} \phi_{i}\right)(1+o) .
\end{aligned}
$$

For the computation of the denominator of $a_{i}$ we have

$$
\begin{aligned}
\mathrm{fl}\left(\left(p_{i}, A p_{i}\right)\right) & =\left(\left(I+D_{i}^{\prime \prime}\right) p_{i},\left(A+E_{i}\right) p_{i}\right)=\left(p_{i}, A p_{i}\right)\left(1+\mu_{i}\right) \\
\left|\mu_{i}\right| & =\left|\left(D_{i}^{\prime \prime} p_{i}, A p_{i}\right)+\left(\left(I+D_{i}^{\prime \prime}\right) p_{i}, E_{i} p_{i}\right)\right| /\left(p_{i}, A p_{i}\right) \\
& \leqq\left\{\varepsilon C_{2}\left\|p_{i}\right\|\left\|A p_{i}\right\|+\varepsilon C_{1}\|A\|\left\|p_{i}\right\|^{2}(1+o)\right\} /\left\|A^{\frac{1}{2}} p_{i}\right\|^{2} \\
& \leqq \varepsilon C_{2} \kappa^{\frac{1}{2}}+\varepsilon C_{1} \kappa(1+o), \quad\left[\varepsilon C_{2} \rightarrow 0\right] .
\end{aligned}
$$

From (3.18) and (3.19) we obtain for the computed $a_{i}$

$$
\begin{aligned}
a_{i}: & =\mathrm{fl}\left(\frac{\mathrm{fl}\left(\left(r_{i}, p_{i}\right)\right)}{\mathrm{fl}\left(\left(p_{i}, A p_{i}\right)\right)}\right)=\frac{\left(\hat{r}_{i}, p_{i}\right)\left(1+\eta_{i}\right)}{\left(p_{i}, A p_{i}\right)\left(1+\mu_{i}\right)}\left(1+\varepsilon_{i}\right) \\
& =\hat{a}_{i}\left(1+\delta a_{i}\right), \\
\hat{a}_{i}: & =\left(\hat{r}_{i}, p_{i}\right) /\left\|A^{\frac{1}{2}} p_{i}\right\|^{2}, \\
\left|\varepsilon_{i}\right| & \leqq \varepsilon \\
\left|\delta a_{i}\right| & =\left|\eta_{i}-\mu_{i}+\varepsilon_{i}+\eta_{i} \varepsilon_{i}\right| /\left|1+\mu_{i}\right| \\
& \leqq \varepsilon\left\{\left(1+C_{2} \kappa^{\frac{1}{2}}+C_{1} \kappa\right)+\alpha_{i}\left(1+C_{2}\right)+C_{1} \alpha_{i} \phi_{i}\right\}(1+o),
\end{aligned}
$$


under the restriction

$$
\varepsilon\left\{\kappa^{\frac{1}{2}}\left(C_{2}+C_{1} \kappa^{\frac{1}{2}}\right)+\alpha_{i}+C_{1} \alpha_{i} \phi_{i}\right\} \rightarrow 0 .
$$

For the computation of $x_{i+1}$ we have

$$
\begin{aligned}
x_{i+1} & =\mathrm{fl}\left(x_{i}+a_{i} p_{i}\right)=\left(I+F_{i}^{\prime \prime}\right)\left(x_{i}+\left(I+F_{i}^{\prime}\right) a_{i} p_{i}\right) \\
& =x_{i}+\hat{a}_{i} p_{i}+\delta x_{i+1}, \\
\delta x_{i+1} & =F_{i}^{\prime \prime} x_{i}+\hat{a}_{i}\left(\delta a_{i} p_{i}+M_{i} p_{i}\right), \\
\left\|M_{i}\right\| & =\left\|\left(1+\delta a_{i}\right)\left(F_{i}^{\prime}+F_{i}^{\prime \prime}\left(I+F_{i}^{\prime}\right)\right)\right\| \leqq 2 \varepsilon(1+o),
\end{aligned}
$$

under the restriction

$$
\varepsilon\left\{\kappa^{\frac{1}{2}}\left(C_{2}+C_{1} \kappa^{\frac{1}{2}}\right)+\alpha_{i}\left(1+C_{2}\right)+C_{1} \alpha_{i} \phi_{i}\right\} \rightarrow 0 .
$$

From the first equalities in (3.22) it follows that

$$
A^{\frac{1}{2}}\left(\hat{x}-x_{i+1}\right)=A^{\frac{1}{2}}\left(\hat{x}-x_{i}\right)-\hat{a}_{i} A^{\frac{1}{2}} p_{i}-A^{\frac{1}{2}} \delta x_{i+1},
$$

and hence, by taking squared norms of both sides,

$$
\begin{aligned}
\left\|A^{\frac{1}{2}}\left(\hat{x}-x_{i+1}\right)\right\|^{2}= & \left\|A^{\frac{1}{2}}\left(\hat{x}-x_{i}\right)-\hat{a}_{i} A^{\frac{1}{2}} p_{i}\right\|^{2} \\
& -2\left(\hat{r}_{i}-\hat{a}_{i} A p_{i}, \delta x_{i+1}\right)+\left\|A^{\frac{1}{2}} \delta x_{i+1}\right\|^{2} .
\end{aligned}
$$

From the definition of $\hat{a}_{i}$ we obtain

$$
\begin{aligned}
\left\|A^{\frac{1}{2}}\left(\hat{x}-x_{i+1}\right)\right\|^{2}= & \left\|A^{\frac{1}{2}}\left(\hat{x}-x_{i}\right)\right\|^{2}-\left(\hat{r}_{i}, p_{i}\right)^{2} /\left\|A^{\frac{1}{2}} p_{i}\right\|^{2} \\
& -2\left(\hat{r}_{i}-\hat{a}_{i} A^{\frac{1}{2}} p_{i}, \delta x_{i+1}\right)+\left\|A^{\frac{1}{2}} \delta x_{i+1}\right\|^{2},
\end{aligned}
$$

which leads to the basic formula

$$
\frac{\left\|A^{\frac{1}{2}}\left(\hat{x}-x_{i+1}\right)\right\|^{2}}{\left\|A^{\frac{1}{2}}\left(\hat{x}-x_{i}\right)\right\|^{2}}=1-\gamma_{i}^{2}\left(1+v_{i+1}\right),
$$

where $\gamma_{i}$ is defined as in (3.10) and

$$
v_{i+1}:=\left\{2\left(\hat{r}_{i}-\hat{a}_{i} A p_{i}, \delta x_{i+1}\right)-\left\|A^{\frac{1}{2}} \delta x_{i+1}\right\|^{2}\right\} /\left\{\hat{a}_{i}\left(\hat{r}_{i}, p_{i}\right)\right\} .
$$

It remains to be proved that $v_{i+1}$ satisfies (3.12) under the restriction (3.13). Note that $\left(\hat{r}_{i}-\hat{a}_{i} A p_{i}, p_{i}\right)=0$ and therefore the term $\hat{a}_{i} \delta a_{i} p_{i}$ in the relation for $\delta x_{i+1}$ of (3.22) cancels when evaluating the inner product in the numerator of (3.28). Consequently, from (3.22) and the definition of $\hat{a}_{i}$ we obtain, evaluating term by term,

$$
\begin{aligned}
& \left|\left(\hat{r}_{i}-\hat{a}_{i} A p_{i}, \delta x_{i+1}\right)\right| /\left|\hat{a}_{i}\left(\hat{r}_{i}, p_{i}\right)\right| \\
& \leqq\left\|\hat{r}_{i}\right\|\left\|F_{i}^{\prime \prime}\right\|\left\|x_{i}\right\|\left\|A^{\frac{1}{2}} p_{i}\right\|^{2} /\left(r_{i}, p_{i}\right)^{2}+\left\|A p_{i}\right\|\left\|F_{i}^{\prime \prime}\right\|\left\|x_{i}\right\| /\left|\left(\hat{r}_{i}, p_{i}\right)\right| \\
& +\left\|\hat{r}_{i}\right\|\left\|M_{i}\right\|\left\|p_{i}\right\| /\left(\hat{r}_{i}, p_{i}\right) \mid+\left\|A p_{i}\right\|\left\|M_{i}\right\|\left\|p_{i}\right\| /\left\|A^{\frac{1}{2}} p_{i}\right\|^{2} \\
& \leqq\left\|F_{i}^{\prime \prime}\right\| \beta_{i}^{2} \phi_{i}+\left\|F_{i}^{\prime \prime}\right\| \beta_{i} \phi_{i}+\left\|M_{i}\right\| \alpha_{i}+\left\|M_{i}\right\| \kappa^{\frac{1}{2}} \\
& \leqq \varepsilon \beta_{i}^{2} \phi_{i}+\varepsilon \beta_{i} \phi_{i}+2 \varepsilon\left(\alpha_{i}+\kappa^{\frac{1}{2}}\right)(1+o) \text {, }
\end{aligned}
$$


under the restriction (3.23). We still have to estimate the second order term in (3.28). This estimate does not affect the numerical constants appearing in the first order terms and therefore we may estimate rather roughly as far as numerical constants are concerned. Using the Cauchy-Schwarz inequality we obtain from (3.20) and (3.22)

$$
\begin{aligned}
&\left\|A^{\frac{1}{2}} \delta x_{i+1}\right\|^{2} /\left|\hat{a}_{i}\left(\hat{r}_{i}, p_{i}\right)\right| \\
& \leqq 3\left\{\left\|F_{i}^{\prime \prime}\right\|^{2}\|A\|\left\|x_{i}\right\|^{2}\left\|A^{\frac{1}{2}} p_{i}\right\|^{2} /\left(\hat{r}_{i}, p_{i}\right)^{2}+\left(\delta a_{i}\right)^{2}\right. \\
&\left.+\left\|M_{i}\right\|^{2}\|A\|\left\|p_{i}\right\|^{2} /\left\|A^{\frac{1}{2}} p_{i}\right\|^{2}\right\} \\
& \leqq 3\left\{\varepsilon^{2} \phi_{i}^{2} \beta_{i}^{2}+3 \varepsilon^{2}\left[3\left(1+C_{2}^{2} \kappa+C_{1}^{2} \kappa^{2}\right)+2 \alpha_{i}^{2}\left(1+C_{2}^{2}\right)\right.\right. \\
&\left.\left.+C_{1}^{2} \alpha_{i}^{2} \phi_{i}^{2}\right]+4 \varepsilon^{2} \kappa\right\}(1+o),
\end{aligned}
$$

under the restriction (3.23). So, finally we obtain from (3.28), (3.29) and (3.30)

$$
\begin{aligned}
\left|v_{i+1}\right| \leqq & 2 \varepsilon\left\{2 \kappa^{\frac{1}{2}}+2 \alpha_{i}+\beta_{i}\left(1+\beta_{i}\right) \phi_{i}\right\}(1+o) \\
& +3 \varepsilon^{2}\left\{9\left(1+C_{2}^{2} \kappa+C_{1}^{2} \kappa^{2}\right)+4 \kappa+6 \alpha_{i}^{2}\left(1+C_{2}^{2}\right)\right. \\
& \left.+\left(\beta_{i}^{2}+3 C_{1}^{2} \alpha_{i}^{2}\right) \phi_{i}^{2}\right\}(1+o),
\end{aligned}
$$

under the restriction (3.23). As this inequality can be written in the more compact form of (3.12), under the restriction (3.13), we have proved Theorem 3.1.

For $\varepsilon \downarrow 0$ the relation (3.11) coincides with relation (2.2) as was to be expected. In Sect. 2 we concluded from relation (2.2) the linear convergence of the natural error to zero for the exact DM under the condition that $\gamma_{i}$ is uniformly bounded away from zero (cf. formula (2.7)). Here, we use (3.11) in order to obtain a similar result for the perturbed DM. From relation (3.11) we conclude that the natural error decreases at the step form $i$ to $i+1$ iff both $v_{i+1}>-1$ and $\gamma_{i}^{2}>0$. We examine both conditions. As far as the condition $\gamma_{i+1}>-1$ is concerned, apparently from (3.12) and (3.13), $\left|v_{i+1}\right|<1$ if the value of

$$
\varepsilon\left\{\kappa^{\frac{1}{2}}\left(1+C_{2}+C_{1} \kappa^{\frac{1}{2}}\right)+\alpha_{i}\left(1+C_{2}\right)+\left(\beta_{i}+\beta_{i}^{2}+C_{1} \alpha_{i}\right) \phi_{i}\right\}
$$

is suitably small. Unfortunately, owing to the use of the $o$-symbol, the formulas (3.12) and (3.13) do not yield explicit bounds for this value to guarantee $\left|v_{i+1}\right|<1$. However, as we said already in subsection 3.1 , one can easily retrace the proof of Theorem 3.1 and replace all $o$-symbols by definite estimates involving explicit numerical constants. For instance, in Bollen [2], pp. 95-96, it is shown that if both

$$
\varepsilon\left\{\kappa^{\frac{1}{2}}\left(1+C_{2}+C_{1} \kappa^{\frac{1}{2}}\right)+\alpha_{i}\left(1+C_{2}\right)\right\} \leqq 1 / 40
$$

and

$$
\varepsilon\left\{2 \beta_{i}\left(1+\beta_{i}\right)+C_{1} \alpha_{i}\right\} \phi_{i} \leqq 1 / 4
$$

then $\left|v_{i+1}\right| \leqq 4 / 5$ and consequently then

$$
\frac{\left\|A^{\frac{1}{2}}\left(\hat{x}-x_{i+1}\right)\right\|^{2}}{\left\|A^{\frac{1}{2}}\left(\hat{x}-x_{i}\right)\right\|^{2}} \leqq 1-\frac{1}{5} \gamma_{i}^{2} .
$$


The restrictions (3.31) and (3.32) were chosen quite arbitrarily and the upper bound $\left|v_{i+1}\right| \leqq 4 / 5$ was obtained by rather rough estimates; they can easily be improved. As far as the equality $\gamma_{i}>0$ is concerned we note that the inequalities of (2.9) also hold in the perturbed case for the parameters $\alpha_{i}, \beta_{i}$ and $\gamma_{i}$ defined in (3.10). Hence, if (3.31) holds, then $\gamma_{i}^{2} \geqq \kappa^{-1} \alpha_{i}^{2} \geqq \kappa^{-1}(40 \varepsilon)^{2}>0$. So, summarizing, if the conditions (3.31) and (3.32) are satisfied then the natural error decreases in the step from $i$ to $i+1$. We now show how these two conditions lead to assertions on the reachable level of the residual for a perturbed DM.

Condition (3.31) contains the fixed quantities $\varepsilon, \kappa, C_{1}$ and $C_{2}$ and the parameter $\alpha_{i}$. The parameter $\alpha_{i}$ depends on the exact residual $\hat{r}_{i}$ after the step form $i-1$ to $i$ and on the (given or computed) machine direction vector $p_{i}$, used in the step from $i$ to $i+1$. For simplicity, we first assume that all direction vectors $p_{i}$ are chosen relational to $\hat{r}_{i}$ in such a way that (3.31) is satisfied in all steps. In comparison with condition (3.31) the condition (3.32) contains extra the parameters $\beta_{i}$ and $\phi_{i}$. The parameter $\beta_{i}$ is bounded at all steps if $\alpha_{i}$ is bounded at all steps. The parameter $\phi_{i}$, however, tends to infinity as $x_{i} \rightarrow \hat{x}$.

Now, if also (3.32) would hold for all steps then it follows from (3.33) that the natural error converges step-wise linearly to zero and consequently then, for $i \rightarrow \infty$, we have $x_{i} \rightarrow \hat{x}, \phi_{i} \rightarrow \infty$ and since $\beta_{i} \geqq \kappa^{-\frac{1}{2}} \alpha_{i} \geqq \kappa^{-\frac{1}{2}}$ this would ultimately contradict (3.32). Therefore, there must be a (first) step, say step $m$, for which (3.32) does not hold. Since the inequality (3.32) is equivalent to the inequality

$$
\left\|b-A x_{i}\right\| \geqq 4 \varepsilon\left\{2 \beta_{i}\left(1+\beta_{i}\right)+C_{1} \alpha_{i}\right\}\|A\|\left\|x_{i}\right\|
$$

it follows that, if (3.31) is satisfied at all steps, then the natural error converges stepwise linearly until the iteration step $m$ where the residual reaches the level

$$
\left\|b-A x_{m}\right\| \leqq 4 \varepsilon\left\{2 \beta_{m}\left(1+\beta_{m}\right)+C_{1} \alpha_{m}\right\}\|A\|\left\|x_{m}\right\| .
$$

In the consecutive steps, $i>m$, the natural error may increase as well as decrease but from every step $i>m$ where (3.32) holds, the natural error converges stepwise linearly until the residual reaches the level (3.34) again, if (3.31) is satisfied at all steps.

If the direction vectors $p_{i}$ are chosen and computed such that (3.31) not necessarily holds for all steps but at least for the steps in which (3.32) holds then the assumption that (3.32) holds for all steps leads to a contradiction in exactly the same way. Therefore, also in that case there is stepwise linear convergence of the natural error until the level represented by (3.34).

Since $\beta_{i} \leqq \alpha_{i}$ inequality (3.34) implies good-behaviour for the perturbed DM on hand, if $\alpha_{i}$ can be bounded by a numerical constant not depending on $\kappa$. In the next section we will see that this is the case for the Gauss-Southwell method (GSM) and the gradient method (GM).

\section{Examples}

In subsections 4.1 and 4.2 we apply the general theory of the Sects. 2 and 3 to the GSM and the GM respectively. In each subsection we first consider the exact method and next the perturbed method. 


\subsection{The Gauss-Southwell Method}

In the exact GSM each direction vector $p_{i}$ is a unit vector $e_{k}$. Therefore, according to (1.2), at each separate step only one component of $x_{i}$ is changed. The coordinate to be changed is chosen such that it corresponds to the largest (in absolute value) component of the (exact) gradient vector $r_{i}:=b-A x_{i}$. Thus, $p_{i}:=e_{k(i)}$, where the index $k(i)(1 \leqq k(i) \leqq n)$ is such that $\left|\left(r_{i}, e_{k(i)}\right)\right| \geqq\left|\left(r_{i}, e_{j}\right)\right|$, for all $1 \leqq j \leqq n$. Since $\|v\|^{2} \leqq n\left(\max \left(v, e_{j}\right)^{2}\right)$, for any real $n$-dimensional vector $v$, we have

$$
\gamma_{i}^{-1} \kappa^{-\frac{1}{2}} \leqq \beta_{i} \leqq \alpha_{i}=\left\|r_{i}\right\|\left\|e_{k(i)}\right\| / \mid\left(r_{i}, e_{k(i)}\right) \| \leqq n^{\frac{1}{2}}
$$

and consequently, from (2.7), it follows that for all $i \geqq 0$ there holds

$$
\left\|A^{\frac{1}{2}}\left(\hat{x}-x_{i+1}\right)\right\| /\left\|A^{\frac{1}{2}}\left(\hat{x}-x_{i}\right)\right\| \leqq\left(1-(n \kappa)^{-1}\right)^{\frac{1}{2}},
$$

for the exact GSM.

For the perturbed GSM the exact residual $\hat{r}_{i}:=b-A x_{i}$ is not available in general, and therefore the coordinate $e_{k(i)}$ to be changed then corresponds to the largest component of the computed residual $r_{i}:=\mathrm{fl}\left(b-\mathrm{fl}\left(A x_{i}\right)\right)$. The inequalities in (4.1) are still valid for the computed residual $r_{i}$ but the equality in (4.1) is not valid since the definition of $\alpha_{i}$ in Theorem 3.1, for the perturbed case, contains the exact residual $\hat{r}_{i}$. We deduce an inequality for $\alpha_{i}$. From the proof of Lemma 3.1 we know that, under the restriction $\varepsilon \rightarrow 0$, we have

$$
r_{i}=\hat{r}_{i}+\delta r_{i}, \quad\left\|\delta r_{i}\right\| /\left\|\hat{r}_{i}\right\| \leqq \varepsilon\left(1+C_{1} \phi_{i}(1+o)\right) .
$$

Hence, again using results of Lemma 3.1, we obtain, under the restriction $\varepsilon\left(1+C_{1} \phi_{i}\right) \rightarrow 0$,

$$
\begin{aligned}
\left(\hat{r}_{i}, e_{k(i)}\right) & =\left(r_{i}, e_{k(i)}\right)\left(1+\xi_{i}\right), \\
\left|\xi_{i}\right| & =\frac{\left(\delta r_{i}, e_{k(i)}\right)}{\left(r_{i}, e_{k(i)}\right)} \leqq \frac{\left\|\delta r_{i}\right\|}{\left\|\hat{r}_{i}\right\|} \frac{\left\|\hat{r}_{i}\right\|}{\left\|r_{i}\right\|} \frac{\left\|r_{i}\right\|}{\left|\left(r_{i}, e_{k(i)}\right)\right|} \\
& \leqq \varepsilon\left(1+C_{1} \phi_{i}\right) \frac{\left\|r_{i}\right\|}{\mid\left(r_{i}, e_{k(i)}\right)}(1+o) \\
& \leqq \varepsilon n^{\frac{1}{2}}\left(1+C_{1} \phi_{i}\right)(1+o) .
\end{aligned}
$$

This implies that $\xi_{i}=o$ under the restriction $\varepsilon n^{\frac{1}{2}}\left(1+C_{1} \phi_{i}\right) \rightarrow 0$. So, finally we obtain,

$$
\begin{aligned}
\alpha_{i} & =\frac{\left\|\hat{r}_{i}\right\|\left\|e_{k(i)}\right\|}{\left|\left(\hat{r}_{i}, e_{k(i)}\right)\right|}=\frac{\left\|r_{i}\right\|\left\|e_{k(i)}\right\|}{\left|\left(r_{i}, e_{k(i)}\right)\right|} \frac{\left\|\hat{r}_{i}\right\|}{\left\|r_{i}\right\|} \frac{\left|\left(r_{i}, e_{k(i)}\right)\right|}{\left|\left(\hat{r}_{i}, e_{k(i)}\right)\right|} \\
& \leqq n^{\frac{1}{2}}(1+o),
\end{aligned}
$$

under the restriction $\varepsilon n^{\frac{1}{2}}\left(1+C_{1} \phi_{i}\right) \rightarrow 0$. Consequently, under the same restriction, we have $\beta_{i} \leqq n^{\frac{1}{2}}(1+o)$ and $\gamma_{i}^{-1} \leqq(n \kappa)^{\frac{1}{2}}(1+o)$.

Retracing the proof of (4.4), using explicit bounds, one can show that, if both $\varepsilon n^{\frac{1}{2}} \leqq 1 / 40$ and $\varepsilon n^{\frac{1}{2}} C_{1} \phi_{i} \leqq 1 / 4$, then $\alpha_{i} \leqq 3 n^{\frac{1}{2}}$ and consequently $\beta_{i} \leqq 3 n^{\frac{1}{2}}$, $\gamma_{i}^{-1} \leqq 3(n \kappa)^{\frac{1}{2}}$. Combining this, (3.31), (3.32) and (3.33) it follows that if both

$$
\varepsilon\left\{\kappa^{\frac{1}{2}}\left(1+C_{2}+C_{1} \kappa^{\frac{1}{2}}\right)+3 n^{\frac{1}{2}}\left(1+C_{2}\right)\right\} \leqq 1 / 40
$$


and

$$
3 \varepsilon\left\{n^{\frac{1}{2}}\left(2+C_{1}\right)+6 n\right\} \phi_{i} \leqq 1 / 4 .
$$

Then we have, for the perturbed GSM,

$$
\frac{\left\|A^{\frac{1}{2}}\left(\hat{x}-x_{i+1}\right)\right\|}{\left\|A^{\frac{1}{2}}\left(\hat{x}-x_{i}\right)\right\|} \leqq\left(1-\frac{1}{45 n \kappa}\right)^{\frac{1}{2}} .
$$

Condition (4.5) only depends on the dimension and the condition number of the linear system, the relative machine precision, the implementation of inner product computations and the implementation of matrix by vector product computations. If (4.5) holds then, similar to the reasoning in subsection 3.2 , assuming that also (4.6) holds at all steps leads to a contradiction. Consequently, rewriting (4.6), we may conclude that if $\left\{x_{i}\right\}$ is generated by the perturbed GSM with an arbitrary initial machine vector $x_{0}$ and if (4.5) is satisfied then the natural error $\left\|A^{\frac{1}{2}}\left(\hat{x}-x_{i}\right)\right\|$ converges stepwise linearly with a convergence ratio no greater than $\left(1-(45 n \kappa)^{-1}\right)^{\frac{1}{2}}$ until the iteration step $m$ where the residual satisfies

$$
\left\|b-A x_{m}\right\| \leqq 12 \varepsilon\left(n^{\frac{1}{2}}\left(2+C_{1}\right)+6 n\right)\|A\|\left\|x_{m}\right\| .
$$

This implies that the GSM is well-behaved and numerically stable if (4.5) is satisfied. The difference between the convergence ratio $\left(1-(n \kappa)^{-1}\right)^{\frac{1}{2}}$ for the exact GSM and the convergence ratio $\left(1-(45 n \kappa)^{-1}\right)^{\frac{1}{2}}$ for the perturbed GSM is caused by the arbitrarily chosen conditions (3.31) and (3.32) and the roughness of the estimates based on these conditions. Of course, a stronger restriction on $\varepsilon$ results in a convergence ratio for the perturbed case which is closer to the convergence ratio for the exact case.

From the fact that for the exact $\operatorname{GSM}\left(r_{i+1}, e_{k(i)}\right)=0(i \geqq 0)$, it even follows that in that case

$$
\left\|A^{\frac{1}{2}}\left(\hat{x}-x_{i+1}\right)\right\| /\left\|A^{\frac{1}{2}}\left(\hat{x}-x_{i}\right)\right\| \leqq\left(1-((n-1) \kappa)^{-1}\right)^{\frac{1}{2}} \quad(i \geqq 1)
$$

but this result is not exploited here.

\subsection{The Gradient Method}

In the exact $\mathrm{GM}$ as successive direction vectors one takes the (exact) residual $p_{i}:=r_{i}:=b-A x_{i}$. We have

$$
\gamma_{i}^{-1} \kappa^{-\frac{1}{2}} \leqq \beta_{i} \leqq \alpha_{i}=\left\|r_{i}\right\|^{2} /\left|\left(r_{i}, r_{i}\right)\right|=1
$$

Consequently, from (2.7), it follows that for all $i \geqq 0$ there holds

$$
\left\|A^{\frac{1}{2}}\left(\hat{x}-x_{i+1}\right)\right\| /\left\|A^{\frac{1}{2}}\left(\hat{x}-x_{i}\right)\right\| \leqq\left(1-\kappa^{-1}\right)^{\frac{1}{2}},
$$

for the exact GM. From the Kantorovich inequality one obtains a sharper bound for $\gamma_{i}$,

$$
\gamma_{i}=\left\|r_{i}\right\|^{2} /\left(\left\|A^{-\frac{1}{2}} r_{i}\right\|\left\|A^{\frac{1}{2}} r_{i}\right\|\right) \geqq 2 \kappa^{\frac{1}{2}} /(\kappa+1)
$$


and hence for all $i \geqq 0$ there even holds

$$
\left\|A^{\frac{1}{2}}\left(\hat{x}-x_{i+1}\right)\right\| / \| A^{\frac{1}{2}}\left(\hat{x}-x_{i}\right) \leqq\left(1-4 \kappa /(\kappa+1)^{2}\right)^{\frac{1}{2}}=(\kappa-1) /(\kappa+1)
$$

for the exact GM.

For the perturbed GM one takes $p_{i}:=r_{i}:=\mathrm{fl}\left(b-\mathrm{fl}\left(A x_{i}\right)\right)$. The inequalities of (4.9) remain valid but instead of the first equality in (4.9) we have $\alpha_{i}$ $=\left\|\hat{r}_{i}\right\|\left\|r_{i}\right\| /\left\|\left(\hat{r}_{i}, r_{i}\right)\right\|$, according to the definition of $\alpha_{i}$ in Theorem 3.1. From Lemma 3.1 it follows that $\alpha_{i}=1+o$, under the restriction $\varepsilon\left(1+C_{1} \phi_{i}\right) \rightarrow 0$, and consequently, $\beta_{i} \leqq 1+o$ and $\gamma_{i}^{-1} \leqq \kappa^{\frac{1}{2}}(1+o)$ under the same restriction. Retracing the proof of Lemma 3.1, using explicit bounds, one can show that if both $\varepsilon \leqq 1 / 40$ and $\varepsilon C_{1} \phi_{i} \leqq 1 / 4$ then $\alpha_{i} \leqq 2$ and consequently $\beta_{i} \leqq 2, \gamma_{i}^{-1} \leqq 2 \kappa^{\frac{1}{2}}$. From this, (3.31), (3.32) and (3.33) it follows that if both

and

$$
\varepsilon\left\{\kappa^{\frac{1}{2}}\left(1+C_{2}+C_{1} \kappa^{\frac{1}{2}}\right)+2\left(1+C_{2}\right)\right\} \leqq 1 / 40
$$

$$
2 \varepsilon\left(6+C_{1}\right) \phi_{i} \leqq 1 / 4
$$

then we have, for the perturbed GM,

$$
\frac{\left\|\dot{A}^{\frac{1}{2}}\left(\hat{x}-x_{i+1}\right)\right\|}{\left\|A^{\frac{1}{2}}\left(\hat{x}-x_{i}\right)\right\|} \leqq\left(1-\frac{1}{20 \kappa}\right)^{\frac{1}{2}} .
$$

From these last three inequalities we can conclude, similar to the general perturbed case and the perturbed GSM, that if $\left\{x_{i}\right\}$ is generated by the perturbed GM with an arbitrary initial machine vector $x_{0}$ and if (4.10) is satisfied then the natural error $\left\|A^{\frac{1}{2}}\left(\hat{x}-x_{i}\right)\right\|$ converges stepwise linearly with a convergence ratio no greater than $\left(1-(20 \kappa)^{-1}\right)^{\frac{1}{2}}$ until the iteration step $m$ where the residual satisfies

$$
\left\|b-A x_{m}\right\| \leqq 8 \varepsilon\left(6+C_{1}\right)\|A\|\left\|x_{m}\right\| .
$$

This implies that the GM is well-behaved and numerically stable if (4.10) is satisfied.

Remark 4.1. One can prove that for the exact GM there holds

$$
\left\|\hat{x}-x_{i+1}\right\| /\left\|\hat{x}-x_{i}\right\| \leqq\left(1-\kappa^{-1}\right)^{\frac{1}{2}} \quad(i \geqq 0),
$$

expressing the step-wise linear convergence of the error with a convergence ratio no greater than $\left(1-\kappa^{-1}\right)^{\frac{1}{2}}$. In Bollen [2] it is proved that if $\left\{x_{i}\right\}$ is generated by the perturbed $\mathrm{GM}$ with an arbitrary initial machine vector $x_{0}$ and if

$$
\varepsilon\left\{\kappa^{\frac{1}{2}}\left(1+C_{2}+C_{1} \kappa^{\frac{1}{2}}\right)+\left(3+C_{2}\right)\right\} \leqq 1 / 40
$$

then the error $\left\|\hat{x}-x_{i}\right\|$ converges step-wise linearly with a convergence ratio no greater than $\left(1-(14 \kappa)^{-1}\right)^{\frac{1}{2}}$ until the iteration step where the natural error satisfies

$$
\left\|A^{\frac{1}{2}}\left(x-x_{m}\right)\right\| \leqq 8 \varepsilon\left(1+\kappa^{\frac{1}{2}}\left(1+2 C_{1}\right)\right)\left\|A^{\frac{1}{2}}\right\|\left\|x_{m}\right\| .
$$




\section{Final Remarks}

Remark 5.1. The Appearance of $C_{1}$ and $C_{2}$. For the same reason as in the perturbed GM case, treated in Bollen [1], final Remark 2, the constants $C_{1}$ and $C_{2}$ do not show up in the first order part of estimate (3.12) for general perturbed DM's. This justifies the rather careful treatment of high order terms, using $o$-symbols. Also the absence of $C_{2}$ in estimate (3.34) for the reachable level can be understood in exactly the same way as is done in Bollen [1] for the perturbed GM. We explain the appearance of $C_{1}$ in estimate (3.34). Assume that during the step from $x_{i}$ to $x_{i+1}$ only round-off occurs at the computation of $A * x_{i}$ and not at the other arithmetical operations like vector addition, inner product computations etc. Then, retracing the proof of Theorem 3.1 we obtain sucessively

$$
\begin{aligned}
\delta r_{i} & =-E_{i} x_{i},\left\|\delta r_{i}\right\| /\left\|\hat{r}_{i}\right\| \leqq \varepsilon C_{1} \phi_{i}, \\
\left|\delta a_{i}\right| & =\left|\eta_{i}\right|=\left|\tau_{i}\right|=\left|\left(\delta r_{i}, p_{i}\right) /\left(\hat{r}_{i}, p_{i}\right)\right| \\
& \leqq\left(\left\|\delta r_{i}\right\| /\left\|\hat{r}_{i}\right\|\right) \alpha_{i} \leqq \varepsilon C_{1} \alpha_{i} \phi_{i}, \quad \delta x_{i+1}=\hat{a}_{i} \delta a_{i} p_{i}, \\
v_{i+1} & =-\left\|A^{\frac{1}{2}} \delta x_{i+1}\right\|^{2} /\left\{\hat{a}_{i}\left(\hat{r}_{i}, p_{i}\right)\right\}=-\left(\delta a_{i}\right)^{2} .
\end{aligned}
$$

Consequently, $\left|v_{i+1}\right| \leqq\left(\varepsilon C_{1} \alpha_{i} \phi_{i}\right)^{2}$, which implies $\left|v_{i+1}\right| \leqq \varepsilon C_{1} \alpha_{i} \phi_{i} o$ under the restriction $\varepsilon C_{1} \alpha_{i} \phi_{i} \rightarrow 0$. This agrees with the appearance of the term containing $\varepsilon C_{1} \alpha_{i} \phi_{i}$ in (3.12) and (3.13) and thus in (3.34). The other terms in (3.12) and (3.13) containing $C_{1}$ are present due to the round-off at the computation of $A * p_{i}$ but they do not influence the reachable level.

Remark 5.2. Recursive Residuals. From Statement (1.2) it follows that for the exact DM one bas $b-A x_{i+1}=b-A x_{i}-a_{i} A p_{i}$ which gives the following recurrence relation for the residual $r_{i+1}=r_{i}-a_{i} A p_{i}$. Hence, one might as well decide to compute $r_{i+1}(i \geqq 0)$ from this relation instead of from relation (1.3). In that case we call the method a recursive residual descent method (RRDM). The vector $A * x_{i}$ is not required anymore and just one matrix by vector product computation is needed per iteration. For the RRDM the sequence $\left\{r_{i}\right\}$ can be computed even without computing the sequence $\left\{x_{i}\right\}$. Of course, in case of exact computations a DM and its corresponding RRDM generate the same sequences $\left\{x_{i}\right\}$ and $\left\{r_{i}\right\}$ when starting with the same initial vector $x_{0}$. However, this is no longer true in the presence of round-off. Under certain conditions the step-wise linear convergence to zero of $\left\|A^{-\frac{1}{2}} r_{i}\right\|$ remains valid for the perturbed RRDM where $\left\{r_{i}\right\}$ are the recursively computed residuals. This follows from Proposition 2.3.1.12 of Bollen [2], p. 62, which states that if $\left\{r_{i}\right\}$ is computed by a perturbed RRDM with an arbitrary initial machine vector $x_{0}$ and if

then

$$
\varepsilon\left\{\kappa^{\frac{1}{2}}\left(1+C_{2}+C_{1} \kappa^{\frac{1}{2}}\right)+\kappa \beta_{i}\left(1+\beta_{i}\right)+\alpha_{i}\left(1+C_{2}+C_{1} \kappa\right)\right\} \leqq 1 / 8
$$

Here,

$$
\left\|A^{-\frac{1}{2}} r_{i+1}\right\|^{2} /\left\|A^{-\frac{1}{2}} r_{i}\right\|^{2} \leqq\left(1-(5 / 16) \gamma_{i}^{2}\right) \quad \text { for all } i \geqq 0 .
$$

$$
\begin{gathered}
\alpha_{i}:=\left\|r_{i}\right\|\left\|p_{i}\right\| /\left|\left(r_{i}, p_{i}\right)\right|, \quad \beta_{i}:=\left\|r_{i}\right\|\left\|A^{\frac{1}{2}} p_{i}\right\| /\left(\left\|A^{\frac{1}{2}}\right\|\left|\left(r_{i}, p_{i}\right)\right|\right), \\
\gamma_{i}:=\left|\left(r_{i}, p_{i}\right)\right| /\left(\left\|A^{-\frac{1}{2}} r_{i}\right\|\left\|A^{\frac{1}{2}} p_{i}\right\|\right) .
\end{gathered}
$$


For the concrete perturbed method RRGM, for instance, where $p_{i}:=r_{i}, \alpha_{i}=1$, $\beta_{i} \leqq 1, \gamma_{i}=\left\|r_{i}\right\|^{2} /\left(\left\|A^{-\frac{1}{2}} r_{i}\right\|\left\|A^{\frac{1}{2}} r_{i}\right\|\right) \geqq 2 \kappa^{\frac{1}{2}}(\kappa+1)$ this means that $\left\|A^{-\frac{1}{2}} r_{i}\right\|$ converges step-wise linearly to zero with a ratio no greater than

$$
\left(1-5 \kappa /(2(\kappa+1))^{2}\right)^{\frac{1}{2}} \quad \text { if } \quad \varepsilon\left\{\left(1+C_{2}\right)+\kappa^{\frac{1}{2}}\left(1+C_{2}\right)+2 \kappa\left(1+C_{1}\right)\right\} \leqq 1 / 8 .
$$

We emphasize that this does not imply that $\left\|A^{\frac{1}{2}}\left(\hat{x}-x_{i}\right)\right\|$ converges step-wise linearly to zero (cf. Bollen [2], Sect. 3.2).

Remark 5.3. Stopping Criterion. Since, in the algorithm one computes already the inner product $\left(r_{i}, p_{i}\right)$ for the computation of $a_{i}$ one can compute straightforwardly an approximation $\tilde{\alpha}_{i}$ of $\alpha_{i}:=\left\|\hat{r}_{i}\right\|\left\|p_{i}\right\| /\left|\left(\hat{r}_{i}, p_{i}\right)\right|$ if one is willing to compute extra $\left\|r_{i}\right\|$ and $\left\|p_{i}\right\|$. If moreover an estimate or upperbound for $\|A\|$ is available then an appropriate upperbound $\tilde{\phi}_{i}$ for $\phi_{i}:=\|A\|\left\|x_{i}\right\| /\left\|\hat{r}_{i}\right\|$ can be obtained straightforwardly by computing $\left\|x_{i}\right\|$ extra. It can easily be verified that these straightforward computations produce an $\tilde{\alpha}_{i}$ which is a reasonable approximation of $\alpha_{i}$ (in relative sense) and a $\tilde{\phi}_{i}$ which is a reasonable upperbound for $\phi_{i}$ as long as $\tilde{\alpha}_{i}$ and $\tilde{\phi}_{i}$ are small in the sense of (3.31) and (3.32). Therefore one might decide to proceed the iterations as long as (3.31) and (3.32) hold for $\tilde{\alpha}_{i}$ and $\tilde{\phi}_{i}$. If one of the two inequalities breaks down then one might stop in case the residual is small enough to guarantee a solution with the described accuracy or one might continue by choosing an alternative direction $p_{i}$. Realize that choosing $p_{i}:=r_{i}$ for all iterations after some step guarantees to reach a residual of the level of the inherent residual. Of course, for well-behaved methods one can use (3.3) as stopping criterion with an appropriate value for $g$. One may ask whether it is possible to verify the monotonicity of the natural error $\left\|A^{\frac{1}{2}}\left(\hat{x}-x_{i}\right)\right\|$ by some computation and use this as a stopping criterion. It is obvious that the objective function

$$
F\left(x_{i}\right)=\left(\hat{x}-x_{i}, A\left(\hat{x}-x_{i}\right)\right)=\left\|A^{\frac{1}{2}}\left(\hat{x}-x_{i}\right)\right\|^{2}
$$

cannot be computed, since the solution vector $\hat{x}$ is not known. On the other hand, however, we have, algebraically,

$$
\begin{aligned}
F\left(x_{i}\right)-F\left(x_{i+1}\right) & =\left(\hat{x}-x_{i}, A\left(\hat{x}-x_{i}\right)\right)-\left(\hat{x}-x_{i+1}, A\left(\hat{x}-x_{i+1}\right)\right) \\
& =\left(x_{i}, A x_{i}\right)-2\left(x_{i}, A \hat{x}\right)-\left(x_{i+1}, A x_{i+1}\right)+2\left(x_{i+1}, A \hat{x}\right) \\
& =\left(x_{i}, A x_{i}\right)-\left(x_{i+1}, A x_{i+1}\right)-2\left(b, x_{i}-x_{i+1}\right)=: G\left(x_{i}, x_{i+1}\right) .
\end{aligned}
$$

This function can be computed and algebraically $F\left(x_{i}\right)>F\left(x_{i+1}\right)$ is equivalent to $G\left(x_{i}, x_{i+1}\right)>0$. The important question rises what can be said if $\mathrm{fl}\left(G\left(x_{i}, x_{i+1}\right)\right)<0$. One can show (cf. Bollen [2], pp. 97, 98), that, due to roundoff, $\mathrm{fl}\left(G\left(x_{i}, x_{i+1}\right)\right)$, computed in the obvious way, can be negative whereas the residual $\left\|b-A x_{i}\right\|$ is still of the order $\varepsilon C_{1} \beta_{i} \kappa\|A\|\left\|x_{i+1}\right\|$. This can be a factor $\kappa$ larger than the reachable level given by (3.34) and therefore then one stops the iterations before the reachable level is attained, if $\mathrm{fl}\left(G\left(x_{i}, x_{i+1}\right)\right)<0$ is used as stopping criterion.

Remark 5.4. Maximal Increase of the Natural Error. For an exact DM, if the direction vector $p_{i}$ is almost orthogonal to the residual $r_{i}$, then $\gamma_{i}$, defined by 
(2.3), is small and consequently, according to (2.2) there will be only a little decrease of the natural error in the next iteration from $x_{i}$ to $x_{i+1}$. Similarly, for a perturbed $\mathrm{DM}$, if the computed direction vector $p_{i}$ is almost orthogonal to the residual $\hat{r}_{i}$, then $\gamma_{i}$ defined in (3.10) is small. Moreover, the parameters $\alpha_{i}$ and $\beta_{i}$ may be to large to guarantee $\left|v_{i+1}\right|<1$ from (3.12). Hence, in view of (3.11) one may ask whether it is even possible for the natural error to increase strongly in the next iteration. To answer this question we write (3.11) in the form

$$
\frac{\left\|A^{\frac{1}{2}}\left(\hat{x}-x_{i+1}\right)\right\|^{2}}{\left\|A^{\frac{1}{2}}\left(\hat{x}-x_{i}\right)\right\|^{2}}=1-\gamma_{i}^{2}+\theta_{i+1},
$$

where, according to (3.11), $\theta_{i+1}:=-\gamma_{i}^{2} v_{i+1}$. From (3.28) it follows that

$$
\theta_{i+1}=\left\{2\left(\hat{a}_{i} A p_{i}-\hat{r}_{i}, \delta x_{i+1}\right)+\left\|A^{\frac{1}{2}} \delta x_{i+1}\right\|^{2}\right\} /\left\|A^{-\frac{1}{2}} \hat{r}_{i}\right\|^{2} .
$$

Estimating term by term in a similar way as is done for $v_{i+1}$ in the proof of Theorem 3.1 yields (cf. Bollen [2], pp. 85-88),

$$
\left|\theta_{i+1}\right| \leqq 4 \varepsilon\left(2 \kappa^{\frac{1}{2}}+\psi_{i}\right)(1+o)+\varepsilon \kappa^{\frac{1}{2}}\left(C_{2}+C_{1} \kappa^{\frac{1}{2}}+C_{1} \psi_{i}\right) o,
$$

under the restriction

$$
\varepsilon\left\{\kappa^{\frac{1}{2}}\left(1+C_{2}+C_{1} \kappa^{\frac{1}{2}}\right)+\left(1+C_{1} \kappa^{\frac{1}{2}}\right) \psi_{i}\right\} \rightarrow 0,
$$

where $\psi_{i}:=\left\|A^{\frac{1}{2}}\right\|\left\|x_{i}\right\| /\left\|A^{-\frac{1}{2}} \hat{r}_{i}\right\|$.

Formulas (5.1), (5.2) and (5.3) indicate that, if $\varepsilon \kappa^{\frac{1}{2}}\left(1+C_{2}+C_{1} \kappa^{\frac{1}{2}}\right)$ is sufficiently small, then, for a perturbed DM, the natural error can never increase significantly as long as the natural error has not reached the level of the inherent natural error, whatever the direction vectors $p_{i}$ might be.

\section{References}

1. Bollen, J.A.M.: Round-off error analysis of the gradient method. To appear in: Banach Center Publications, volume: Computational Mathematics (to appear)

2. Bollen, J.A.M.: Round-off error analysis of descent methods for solving linear equations. Doctoral dissertation, Eindhoven University of Technology, 1980

3. Bulirsch, R., Stoer, J.: Introduction to Numerical Analysis. New York: Springer 1979

4. Woźniakowski, H.: Round-off error analysis of the Chebyshev method for the solution of large linear systems. Numer. Math. 2, 191-209 (1977)

5. Woźniakowski, H.: Round-off error analysis of iterations for large linear systems. Numer. Math. 30, 301-314 (1978) 